TRANSACTIONS OF THE

AMERICAN MATHEMATICAL SOCIETY

Volume 352, Number 8 , Pages 3687-3706

S 0002-9947(00)02451-X

Article electronically published on March 16, 2000

\title{
PARTIAL DIFFERENTIAL EQUATIONS WITH MATRICIAL COEFFICIENTS AND GENERALIZED TRANSLATION OPERATORS
}

\author{
N. H. MAHMOUD
}

Abstract. Let $\Delta_{\alpha}$ be the Bessel operator with matricial coefficients defined on $(0, \infty)$ by

$$
\Delta_{\alpha} U(t)=U^{\prime \prime}(t)+\frac{2 \alpha+I}{t} U^{\prime}(t)
$$

where $\alpha$ is a diagonal matrix and let $q$ be an $n \times n$ matrix-valued function. In this work, we prove that there exists an isomorphism $X$ on the space of even $\mathcal{C}^{\infty}, \mathbb{C}^{n}$-valued functions which transmutes $\Delta_{\alpha}$ and $\left(\Delta_{\alpha}+q\right)$. This allows us to define generalized translation operators and to develop harmonic analysis associated with $\left(\Delta_{\alpha}+q\right)$. By use of the Riemann method, we provide an integral representation and we deduce more precise information on these operators.

\section{INTRODUCTION}

Let $\left(\Delta_{\alpha}+q\right)$ be the perturbed Bessel operator with matricial coefficients defined, on $] 0, \infty[$, by

$$
\left(\Delta_{\alpha}+q\right) U(t)=U^{\prime \prime}(t)+\frac{2 \alpha+I}{t} U^{\prime}(t)+q(t) U(t),
$$

where $U$ and $q$ are matrix-valued functions, and $\alpha$ denotes the $n$ order diagonal matrix

$$
\alpha=\left[\begin{array}{ccc}
\alpha_{1} & & 0 \\
& \ddots & \\
0 & & \alpha_{n}
\end{array}\right]
$$

with $\alpha_{n} \geq \ldots \geq \alpha_{1}>-\frac{1}{2}$.

It is well known (see [20]) that the radial Schrödinger equation with coupling between $l^{t h}$ and the $(l+2)^{n d}$ angular momentum gives us a system of singular equations which can be associated with an operator in the form (1.1).

The first goal of this paper is to establish the existence of an operator $X$ (transmutation operator) which transmutes $\left(\Delta_{\alpha}+q\right)$ and $\Delta_{\alpha}$, in the sense

$$
\left(\Delta_{\alpha}+q\right) X=X \Delta_{\alpha}
$$

Received by the editors July 30, 1996 and, in revised form, January 30, 1998.

2000 Mathematics Subject Classification. Primary 35A25, 35C15; Secondary 34 B30.

Key words and phrases. Singular differential operators, Bessel functions, transmutation operators, generalized translations, Riemann function, product formula.

(C)2000 American Mathematical Society 
and then reduces the study of $\left(\Delta_{\alpha}+q\right)$ to that of $\Delta_{\alpha}$. This will be done thanks to an integral representation of the regular solution of the following differential equation:

$$
U^{\prime \prime}(t)+\frac{I+2 \alpha}{t} U^{\prime}(t)+q(t) U(t)+\lambda^{2} U(t)=0, \quad \lambda \in \mathbb{C},
$$

in terms of Bessel functions.

The second part is devoted to the study of generalized translation operators $T_{y}, y \in \mathbb{R}$, associated with $\left(\Delta_{\alpha}+q\right)$. This follows the framework of J. L. Lions [16] and generalizes some results known in the scalar case (see [6] and [22]). We use two techniques to study these operators; the first one is the Fourier transform associated with the spectral decomposition of $\left(\Delta_{\alpha}+q\right)$. We will see that if $f$ is a $\mathcal{C}^{\infty}$ matrix-valued function with compact support, then the function $u(x, y)=T_{y} f(x)$ is a solution of the Cauchy problem

$$
\left\{\begin{array}{l}
\left(\Delta_{\alpha}+q\right)_{x} u(x, y)=\left(\Delta_{\alpha}+q\right)_{y}^{*} u(x, y) \\
u(x, 0)=f(x),\left.\frac{\partial u}{\partial y}\right|_{(x, 0)}=0
\end{array}\right.
$$

where

$$
\left(\Delta_{\alpha}+q\right)^{*} U(t)=U^{\prime \prime}(t)+U^{\prime}(t) \frac{2 \alpha+I}{t}+U(t) q^{*}(t),
$$

and $q^{*}(t)$ is the conjugate of the matrix $q(t)$.

This leads to the second technique which consists in solving (1.3). The Riemann method seems to be convenient for our purpose. Combining these two ways we deduce some properties of the generalized translation operators. Among others we deduce the product formula for the regular solution of (1.2).

The Riemann method has been used by B. L. J. Braaksma and H. S. V. De Snoo and others (see [2], [5], 7]) to solve the scalar Cauchy problem associated with (1.3), and by $\mathrm{Coz}$ (see [10]) to study the kernel of the transmutation operator associated with a singular second order differential operator with matricial coefficients.

I am grateful to Professor Houcine Chebli for instructive conversations and helpful suggestions.

\section{NOTATION AND HyPOTHESES}

Let $M_{n}(\mathbb{C})$ be the space of square $n$ order complex matrices and $I$ the identity matrix. The conjugate of a matrix $A$ in $M_{n}(\mathbb{C})$ is denoted by $A^{*}$. The norm of $A=\left(a_{j k}\right)_{1 \leq j, k \leq n}$ will be defined by

$$
\|A\|=\max _{1 \leq j \leq n} \sum_{1 \leq k \leq n}\left|a_{j k}\right| .
$$

Let $f$ be a holomorphic matrix-valued function. For any bounded domain $D \subset \mathbb{C}$ we denote the norm of $f$ on $D$ by

$$
\|f\|_{D}=\sup _{z \in D}\|f(z)\| .
$$

Let $J_{\nu}$ and $H_{\nu}^{(1)}$ be the Bessel functions of the first and the third kind of index $\nu, \nu \in \mathbb{R}$ (see [22]). We denote by $\Gamma(\alpha), J_{\alpha}$ and $H_{\alpha}^{(1)}$ the diagonal matrix-valued functions defined for, $1 \leq k \leq n$, by

$$
[\Gamma(\alpha)]_{k k}=\Gamma\left(\alpha_{k}\right), \quad\left[J_{\alpha}\right]_{k k}=J_{\alpha_{k}}, \quad \text { and } \quad\left[H_{\alpha}^{(1)}\right]_{k k}=H_{\alpha_{k}}^{(1)} .
$$


Let $\mathcal{J}_{\alpha+p}, p \geq 0$, and $j_{\alpha}$ be the diagonal matrix-valued functions defined, for $t>0$ and $\lambda \in \mathbb{C}$, by

$$
\mathcal{J}_{\alpha+p}(\lambda, t)=2^{\alpha} \Gamma(\alpha+I) \lambda^{-\alpha-p I} J_{\alpha+p I}(\lambda t), j_{\alpha}(\lambda t)=t^{-\alpha} \mathcal{J}_{\alpha}(\lambda, t) .
$$

Let $G(t)$ be the $M_{n}(\mathbb{C})$-valued function defined on [ $0, \infty[$ by

$$
G(t)=\left[\begin{array}{lll}
t^{\alpha_{1}+\frac{1}{2}} & & 0 \\
& \ddots & \\
0 & & t^{\alpha_{n}+\frac{1}{2}}
\end{array}\right],
$$

so the operator $\left(\Delta_{\alpha}+q\right)$ can be written in the form

$$
\left(\Delta_{\alpha}+q\right) U(t)=G^{-1}(t)\left[G U^{\prime}\right]^{\prime}(t)+q(t) U(t) .
$$

The transformation $v=G U$ carries the operator $\left(\Delta_{\alpha}+q\right)$ into the perturbed Bessel operator $L^{Q}$ given by

$$
L^{Q} v(t)=v^{\prime \prime}(t)+\left[\frac{\frac{I}{4}-\alpha^{2}}{t^{2}}+Q(t)\right] v(t), \quad t>0 .
$$

The notions of continuity, differentiability, etc., when applied to matrix-valued functions, are always meant to hold for each coefficient. For $Y$ and $Z$ two differentiable matrix functions, we denote by $\mathcal{W}[Y, Z]$ their Wronskian

$$
\mathcal{W}[Y, Z](t)=Y^{*}(t) G^{2}(t) Z^{\prime}(t)-Y^{\prime *}(t) G^{2}(t) Z(t),
$$

which is independent of $t$ if both $Y$ and $Z$ satisfy (1.2).

We need the following hypotheses:

$\left(\mathbf{A}_{\mathbf{1}}\right) \quad q$ is even and holomorphic on $\mathbb{C}$.

$\left(\mathbf{A}_{2}\right) \quad Q(t)=\left[G q G^{-1}\right](t), t>0$, is an hermitian matrix.

$\left(\mathbf{A}_{\mathbf{3}}\right) \sigma(t)=\int_{t}^{\infty} s\|Q(s)\| d s<+\infty, t>0$

\section{Asymptotic Behavior}

3.1. Behavior at zero. We consider the class of singular second order differential equations

$$
L^{Q} v(t)+\lambda^{2} v(t)=0, \quad t>0,
$$

where $\lambda$ is a real or complex parameter.

Thanks to an idea introduced by [13] (see also [18]) we show that the series

$$
\Phi_{\lambda}(t)=G^{-1}(t)\left[\sum_{p \geq 0} t^{p+\frac{1}{2}} B_{p}(t) \mathcal{J}_{\alpha+p}(\lambda, t)\right] G(t)
$$

is a formal solution of (3.1) provided that the coefficients $B_{p}, p \geq 0$, satisfy the recursive process

$$
\begin{cases}{\left[G^{-1}(t) B_{0}(t) G(t)\right]^{\prime}} & =0 \\ {\left[t^{p+1} G^{-1}(t) B_{p+1}(t) G(t)\right]^{\prime}} & =-\frac{t^{p}}{2} G^{-1}(t)\left[\left(\Delta_{-\alpha}+q^{*}\right) B_{p}\right](t) G(t) .\end{cases}
$$

Let us choose $B_{0}(t)=I$. Our purpose is to define $B_{p}, p \geq 1$, by the same mean as in [18]; we need the following lemma. 
Lemma 3.1. Under the hypotheses $\left(\mathrm{A}_{1}\right)$ and $\left(\mathrm{A}_{2}\right)$, for $p \in \mathbb{N}$, the matrix functions $B_{p+1}(t)$ and $t^{2 p} G^{-2}(t) B_{p+1}(t) G^{2}(t)$, where $B_{p+1}$ is defined by

$$
B_{p+1}(t)=-\frac{1}{2 t^{p+1}} G(t) \int_{0}^{t} u^{p} G^{-1}(u)\left[\left(\Delta_{-\alpha}+q^{*}\right) B_{p}\right](u) G(u) d u G^{-1}(t),
$$

are even and analytic.

Proof. From the hypotheses $\left(\mathrm{A}_{1}\right)$ and $\left(\mathrm{A}_{2}\right)$ we deduce that, for $1 \leq i, j \leq n$, we have $\alpha_{i}-\alpha_{j} \in \mathbb{Z}$. The hypothesis $\left(\mathrm{A}_{2}\right)$ can be expressed in the form

$$
u^{-\alpha_{i}+\alpha_{j}} \bar{q}_{j i}(u)=u^{\alpha_{i}-\alpha_{j}} q_{i j}(u)
$$

then we define $B_{1}$ by

$$
\begin{aligned}
{\left[B_{1}\right]_{i j}(t) } & =-\frac{1}{2 t^{-\alpha_{i}+\alpha_{j}+1}} \int_{0}^{t} u^{-\alpha_{i}+\alpha_{j}} \bar{q}_{j i}(u) d u, \quad i \leq j, \\
& =-\frac{1}{2 t^{-\alpha_{i}+\alpha_{j}+1}} \int_{0}^{t} u^{\alpha_{i}-\alpha_{j}} q_{i j}(u) d u, \quad i \geq j
\end{aligned}
$$

We deduce that the matrix functions $B_{1}(t)$ and $G^{-2}(t) B_{1}(t) G^{2}(t)$ are even and analytic and recursively we obtain the lemma.

Proposition 3.2. Suppose that $q$ is holomorphic in the disc $D(0, R)$. Then

i) For $k, p \geq 0$, the functions $B_{p}$, defined by the recursive formula (3.3), satisfy

$$
\left\|B_{p+p_{0}}^{(k)}\right\|_{D(0, R)} \leq \frac{k ! p^{k}}{2^{p-1} R^{k} p !}\left[\frac{2 c p^{2}}{R^{2}}+M\right]^{p-1}\left\|B_{p_{0}}\right\|_{D(0, R)}
$$

where $c=\|I-2 \alpha\|, M=\|q\|_{D(0, R)}$ and $p_{0}=\alpha_{n}-\alpha_{1}$.

ii) The infinite series (3.2) is uniformly convergent on every compact subinterval of $] 0,1+\|I-2 \alpha\|^{-1 / 2} \operatorname{Re}^{-1}\left[\right.$; its sum $\Phi_{\lambda}(t)$ is an $n$ order matrix whose rows are vector solutions of (3.1), and which satisfies the asymptotic relation

$$
\Phi_{\lambda}(t)=G(t)[I+o(1)], \quad \lim _{t \rightarrow 0^{+}} o(1)=0 .
$$

Proof. Since (3.3) can be written as

$$
\left[B_{p+1}\right]_{i j}(t)=-\frac{1}{2 t^{\alpha_{j}-\alpha_{i}+p+1}} \int_{0}^{t} u^{\alpha_{j}-\alpha_{i}+p}\left[\left(\Delta_{-\alpha}+q^{*}\right) B_{p}\right]_{i j}(u) d u, \quad 1 \leq i, j \leq n
$$

then, for $p+\alpha_{j}-\alpha_{i} \geq 0$, we use estimates of [18, p. 264], and the proposition is deduced.

Corollary 3.3. The equation (1.1) has an unique solution $\psi(\lambda, t)=G^{-1}(t) \Phi_{\lambda}(t)$ satisfying the following asymptotic behavior:

$$
\lim _{t \rightarrow 0} \psi(\lambda, t)=I
$$

Using the Sonine integral representation of the Bessel function of the first kind $J_{\alpha}$ (see [22]), we deduce:

Corollary 3.4. The function $\psi$ has the Sonine type integral representation

$$
\psi(\lambda, t)=j_{\alpha}(\lambda t)+\int_{0}^{t} M(t, u) G^{2}(u) j_{\alpha}(\lambda u) d u
$$


where $j_{\alpha}$ is given by (2.1) and

$$
M(t, u)=t G^{-2}(t) \sum_{1}^{\infty} \frac{B_{p}(t)\left(t^{2}-u^{2}\right)^{p-1}}{2^{p-1} \Gamma(p)},
$$

the series being uniformly convergent on every compact subinterval of $(0, \infty)$.

3.2. Behavior at infinity. Let us denote by $\mathcal{H}_{\alpha}^{(1)}$ the diagonal matrix defined by

$$
\mathcal{H}_{\alpha}^{(1)}(t)=\sqrt{\frac{\pi t}{2}} e^{i\left(\alpha+\frac{I}{2}\right) \frac{\pi}{2}} H_{\alpha}^{(1)}(t),
$$

where $H_{\alpha}^{1}$ is given by the formula $(2.1)$.

Theorem 3.5. i) For $\lambda \in \mathbb{C}, \lambda \neq 0, t>0$, the differential equation (3.1) has a fundamental system of solutions $F(\lambda, t)$ and $F(-\lambda, t)$ satisfying the following asymptotic behavior:

$$
F(\lambda, t)=e^{-i \lambda t}\left[I+o\left(\frac{1}{|\lambda| t}\right)\right], \quad \lim _{|\lambda| t \rightarrow+\infty} o\left(\frac{1}{|\lambda| t}\right)=0 .
$$

ii) The mapping $\lambda \mapsto F(\lambda, t)$ is analytic in $\{\lambda \in \mathbb{C}, \Im m \lambda<0\}$ and continuous in $\{\lambda \in \mathbb{C}, \Im m \lambda \leq 0\}$.

Proof. Using the method of variation of parameters in the equation

$$
v^{\prime \prime}(t)+\frac{\frac{I}{4}-\alpha^{2}}{t^{2}} v(t)+\lambda^{2} v(t)=-Q(t) v(t), \quad t>0,
$$

we obtain the following integral equation:

$$
F(\lambda, t)=\mathcal{H}_{\alpha}^{(1)}(\lambda t)-\int_{t}^{\infty} K\left(\lambda, t, t^{\prime}\right) Q\left(t^{\prime}\right) F\left(\lambda, t^{\prime}\right) d t^{\prime}
$$

where the kernel $K\left(\lambda, t, t^{\prime}\right)$ is defined by

$$
K\left(\lambda, t, t^{\prime}\right)=\frac{e^{2 i \alpha \pi}}{2 i \lambda}\left[\mathcal{H}_{\alpha}^{(1)}\left(\lambda t^{\prime}\right) \mathcal{H}_{\alpha}^{(1)}(-\lambda t)-\mathcal{H}_{\alpha}^{(1)}(\lambda t) \mathcal{H}_{\alpha}^{(1)}\left(-\lambda t^{\prime}\right)\right]
$$

Using estimates on $\mathcal{H}_{\alpha}^{(1)}$, the hypothesis $\left(\mathrm{A}_{3}\right)$ and successive approximations we deduce the existence of $F(\lambda, t)$ and its behavior at infinity (see similar results in 4], [20]).

Corollary 3.6. i) Let $0<t_{0} \leq t$; for $\lambda \in \mathbb{C}$, ऽm $\lambda \leq 0$, and $|\lambda| \geq \lambda_{0}>0$, we have

$$
F^{\prime}(\lambda, t)=-i \lambda e^{-i \lambda t}\left[I+o\left(\frac{1}{|\lambda| t}\right)\right], \quad \lim _{|\lambda| t \rightarrow+\infty} o\left(\frac{1}{|\lambda| t}\right)=0 .
$$

ii) For $\Im m \lambda<0$, we have

$$
\lim _{|\lambda| t \rightarrow+\infty} \dot{F}(\lambda, t)=0, \quad \lim _{|\lambda| t \rightarrow+\infty} \dot{F}^{\prime}(\lambda, t)=0 .
$$


3.3. The $c$-matrix. Let $E(\lambda, t)=G^{-1}(t) F(\lambda, t)$ be the solution of (1.2) associated with $F(\lambda, t)$. Using the known fact that the Wronskian of $\psi(\lambda, t)$ and $E(-\bar{\lambda}, t)$ is independent of the variable $t$, we set

$$
c(\lambda)=\frac{1}{2 i \lambda} \mathcal{W}[E(-\bar{\lambda}, t), \psi(\lambda, t)], \quad \lambda \neq 0 .
$$

This matrix, the analogue of the Harish-Chandra $c$-function, intervenes in the density of the spectral measure associated with the operator $\left(\Delta_{\alpha}+q\right)$. The properties of $\psi(\lambda, t)$ and $E(\lambda, t)$ allow us to deduce the following results.

Proposition 3.7. i) The mapping $\lambda \mapsto c(\lambda)$ is analytic for $\Im m \lambda<0$, and

$$
c(\lambda)=\sqrt{\frac{1}{2 \pi}} 2^{\alpha} \Gamma(\alpha) e^{-i\left(\alpha+\frac{I}{2}\right) \frac{\pi}{2}} G^{-1}(\lambda)\left[I+o\left(\frac{1}{|\lambda|}\right)\right], \quad \lim _{|\lambda| \rightarrow \infty} o\left(\frac{1}{|\lambda|}\right)=0 .
$$

ii) There exists a constant $N>0$ such that, for any $\lambda \in \mathbb{C}, \Im m \lambda \leq 0,|\lambda| \geq$ $N ; c(\lambda)$ is invertible. The mapping $\lambda \mapsto c^{-1}(\lambda)$ is a meromorphic function on $\Im m \lambda<0$ with simple poles.

Proof for similar results are given in [17].

\section{FouRIER TRANSFORM}

Let $\mathcal{D}$ be the space of even $\mathcal{C}^{\infty}, \mathbb{C}^{n}$-valued functions with compact support.

Definition 4.1. For every $\mathrm{f}$ in $\mathcal{D}$ we define the generalized Fourier transform $\mathcal{F}(f)$, associated with $\left(\Delta_{\alpha}+q\right)$, by

$$
\mathcal{F}(f)(\lambda)=\int_{0}^{\infty} \psi^{*}(-\bar{\lambda}, t) G^{2}(t) f(t) d t .
$$

Remark 4.2. The Fourier-Bessel transform $\mathcal{F}_{\alpha}$, associated with $\Delta_{\alpha}$, is a $\mathbb{C}^{n}$-valued operator defined for $\mathrm{f} \in \mathcal{D}$ by

$$
\left\{\mathcal{F}_{\alpha}(f)\right\}_{k}(\lambda)=\int_{0}^{\infty} j_{\alpha_{k}}(\lambda u) u^{2 \alpha_{k}+1} f_{k}(u) d u, \quad 1 \leq k \leq n .
$$

Let $L_{2}^{G}$ be the Hilbert space defined by

$$
L_{2}^{G}=\{f:] 0, \infty\left[\rightarrow \mathbb{C}^{n} \mid\|f\|_{2}^{2}=\int_{0}^{\infty} f^{*}(x) G^{2}(x) f(x) d x<+\infty\right\} .
$$

The Kato-Rellich criterion shows that the operator $\left(\Delta_{\alpha}+q, \mathcal{D}\right)$ is an unbounded essentially self adjoint operator in $L_{2}^{G}$. Its absolute continuous spectrum, parametrized by $\lambda$, is equal to $[0, \infty[$. The discrete spectrum is composed with a finite number of negative eigenvalues: $-\lambda_{1}^{2}, \ldots,-\lambda_{m}^{2}$, where the $\lambda_{j}, 1 \leq j \leq m$, are zeros of $\lambda \rightarrow \operatorname{detc}(\lambda)$. Let $R_{j}$ be the residue of $c^{-1}(\lambda)$ at $\lambda_{j}$; then $\psi\left(\lambda_{j}, t\right) R_{j}$ is the associated eigenfunction.

Let $S_{j}$ be the inverse matrix of

$$
\int_{0}^{\infty}\left[\psi\left(\lambda_{j}, t\right) R_{j}\right]^{*} G^{2}(t) \psi\left(\lambda_{j}, t\right) R_{j} d t,
$$

and let $S$ be the matrix defined, for $\lambda \neq \lambda_{j}$, by

$$
S^{-1}(\lambda)=2 \pi c^{*}(\lambda) c(\lambda) .
$$


The properties of $c(\lambda)$ and its asymptotic behavior allow us to deduce that $S(\lambda)$ is a tempered measure and that

$$
S(\lambda)=S^{*}(\lambda)=S(-\lambda) .
$$

To obtain the spectral theorem we shall introduce some new spaces (see [12]). Let $L_{2}^{S}$ be the Hilbert space of $\mathbb{C}^{n}$-valued functions $f$ defined by

$$
L_{2}^{S}=\{f:] 0, \infty\left[\rightarrow \mathbb{C}^{n} \mid\|f\|_{S}^{2}=\int_{0}^{\infty} f^{*}(\lambda) S(\lambda) f(\lambda) d \lambda<+\infty\right\} .
$$

For $u$ and $v$ two elements of $\left(\mathbb{C}^{n}\right)^{m}$ we put

$$
\langle\langle u, v\rangle\rangle=\sum_{j=1}^{m} v_{j}^{*} S_{j} u_{j} .
$$

Finally let $\widehat{\mathcal{H}}=L_{2}^{S} \oplus\left(\mathbb{C}^{n}\right)^{m}$, and

$$
\begin{aligned}
& H=\left\{g: \mathbb{C} \rightarrow \mathbb{C}^{n}, \text { even, analytic } \mid\right. \exists R>0, \forall k \in \mathbb{N}, \\
& \sup _{\lambda \in \mathbb{C}}\left[\left(1+|\lambda|^{2}\right)^{k} e^{-R|\Im m \lambda|}\|g(\lambda)\|<+\infty\right\} .
\end{aligned}
$$

Making use of the spectral theory of self adjoint operators on Hilbert spaces (see [11) and the last results we deduce the following

Theorem 4.3. i) (Inversion Formula) The mapping $f \mapsto \mathcal{F}(f)$ extends to an isometric isomorphism from $L_{2}^{G}$ onto $\widehat{\mathcal{H}}$; the inverse mapping is given by

$$
f(t)=\int_{0}^{\infty} \psi(\lambda, t) S(\lambda) \mathcal{F}(f)(\lambda) d \lambda+\sum_{j=1}^{m} \psi\left(\lambda_{j}, t\right) R_{j} S_{j} \hat{f}_{j},
$$

with

$$
\hat{f}_{j}=\int_{0}^{\infty}\left[\psi\left(\lambda_{j}, s\right) R_{j}\right]^{*} G^{2}(s) f(s) d s .
$$

ii) (Plancherel Formula) For any $f$ in $L_{2}^{G}$ we have

$$
\|f\|_{2}^{2}=\|\mathcal{F}(f)\|_{S}^{2}+\sum_{j=1}^{m} \hat{f}_{j}^{*} S_{j} \hat{f}_{j} .
$$

iii) (Paley-Wiener type theorem) The mapping $f \mapsto \mathcal{F}(f)$ is a bijection from $\mathcal{D}$ onto $H$.

\section{Transmutation}

Let $C^{2}$ be the space of twice continuous differentiable $\mathbb{C}^{n}$-valued functions defined on ]0, $\infty$. Let $C_{*}$ be the space of even $C^{\infty}, \mathbb{C}^{n}$-valued functions, equipped with the topology of the uniform convergence, on every compact subset of $\mathbb{R}$, of the functions and their derivatives. Let $\mathcal{E}$ be the subspace of functions in $C^{2}$ such that $G^{-1} f \in C_{*}$.

Let us recall (see [18) that the operator $\mathcal{X}$ defined on $\mathcal{E}$ by

$$
\mathcal{X} f(t)=f(t)+G(t) \int_{0}^{t} M(t, u) G(u) f(u) d u, \quad t>0,
$$


where $M(t, u)$ is given by the formula (3.7), is a permutation operator between $L^{Q}$ and $L^{0}$, that is,

$$
L^{Q} \mathcal{X}=\mathcal{X} L^{0} .
$$

5.1. Transmutation associated with $\left(\Delta_{\alpha}+q\right)$. Since the operator $\left(\Delta_{\alpha}+q\right)$ is related to $L^{Q}$ by the formula

$$
\left(\Delta_{\alpha}+q\right)=G^{-1} L^{Q} G,
$$

let us consider the operator $X$ defined by

$$
X=G^{-1} \mathcal{X} G
$$

then the formulas (5.1) and (5.2) allow us to deduce that for any $h$ in $\mathcal{D}$

$$
\left(\Delta_{\alpha}+q\right) X h=X \Delta_{\alpha} h,
$$

with

$$
X h(t)=h(t)+\int_{0}^{t} M(t, u) G^{2}(u) h(u) d u, \quad t>0 .
$$

Theorem 5.1. The operator $X$ is an isomorphism on the space $C_{*}$ and for any $h$ in $C_{*}$ we have

i) $\lim _{t \rightarrow 0} X h(t)=h(0)$,

ii) $X \Delta_{\alpha} h=\left(\Delta_{\alpha}+q\right) X h$.

Proof. Let us consider the integral equation of Gelfand-Levitan type

$$
g(x)=h(x)+\int_{0}^{x} M(x, u) G^{2}(u) h(u) d u, \quad x>0 .
$$

Lemma (3.1) and Proposition 3.2 lead that the kernel $M(x, u) G^{2}(u)$ is continuous for $0 \leq u \leq x$, so the previous integral equation has an unique solution which is continuous. To deduce i) we note, using the Proposition 3.2 that

$$
X h(t)=h(t)+\sum_{0}^{\infty} \frac{G^{-2}(t) t^{2 p+2} B_{p+1}(t) G^{2}(t)}{2^{p} p !} \int_{0}^{1}\left(1-v^{2}\right)^{p} G^{2}(v) h(t v) d v .
$$

To have ii) we take $f=G h, h \in C_{*}$ and we use the formulas (5.2), (5.3) and (5.4).

Definition 5.2. We denote by $C_{*}^{\prime}$ the space of even $\mathbb{C}^{n}$ valued distributions with compact support.

Proposition 5.3. The operator ${ }^{t} X$ defined on $C_{*}^{\prime}$ by

$$
\left\langle{ }^{t} X T, g\right\rangle=\langle T, X g\rangle, \quad g \in C_{*},
$$

is an isomorphism on $C_{*}^{\prime}$.

Proof. It is an immediate consequence of Theorem 5.1.

Corollary 5.4. For any $g$ in $\mathcal{D},{ }^{t} X(g)$ is given by

$$
{ }^{t} X g(x)=g(x)+\int_{x}^{\infty} M^{*}(u, x) G^{2}(u) g(u) d u, \quad x>0 .
$$

The function ${ }^{t} X(g)$ is even and continuous with compact support. 


\subsection{Generalized Fourier transform and transmutation.}

Lemma 5.5. For any $f$ in $\mathcal{D}, X f$ is in $\mathcal{F}^{-1}\left(L_{2}^{S}\right)$.

Proof. For $f$ in $\mathcal{D}$, we have the following Fourier-Bessel inverse formula, associated with $\Delta_{\alpha}$

$$
f(t)=\frac{1}{2 \pi} \int_{0}^{\infty} j_{\alpha}(t \lambda) G^{2}(\lambda) \mathcal{F}_{\alpha} f(\lambda) d \lambda,
$$

where $j_{\alpha}$ is given by (2.2). Using (3.6) and the last formula, we deduce that

$$
\begin{aligned}
X f(t) & =\frac{1}{2 \pi} \int_{0}^{\infty} \psi(\lambda, t) G^{2}(\lambda) \mathcal{F}_{\alpha} f(\lambda) d \lambda \\
& =\frac{1}{2 \pi} \int_{0}^{\infty} \psi(\lambda, t) S(\lambda)\left[S^{-1}(\lambda) G^{2}(\lambda) \mathcal{F}_{\alpha} f(\lambda)\right] d \lambda
\end{aligned}
$$

so we deduce the lemma.

The following proposition allows us to link the operators $\mathcal{F}$ and $\mathcal{F}_{\alpha}$ using the transmutation operator $X$.

Proposition 5.6. For any $f$ in $\mathcal{D}$ we have

$$
\begin{aligned}
& \mathcal{F} X f(\lambda)=c^{*}(\lambda) c(\lambda) G^{2}(\lambda) \mathcal{F}_{\alpha} f(\lambda), \quad \lambda \in \mathbb{C}, \\
& \mathcal{F}(f)=\mathcal{F}_{\alpha}{ }^{t} X(f) .
\end{aligned}
$$

Proof. Formula (5.8) is a consequence of the previous lemma and (4.3).

The operators $\mathcal{F}$ and $\mathcal{F}_{\alpha}$ are isometries so that for $f$ and $g$ in $\mathcal{D}$ we have

$$
\langle X f, g\rangle_{2}=\langle\mathcal{F} X f, \mathcal{F} g\rangle_{\widehat{\mathcal{H}}},
$$

and

$$
\left\langle f,{ }^{t} X(g)\right\rangle_{2}=\left\langle\mathcal{F}_{\alpha}(f), \mathcal{F}_{\alpha}{ }^{t} X(g)\right\rangle_{2}
$$

using the formula (5.8) we show that

$$
\langle\mathcal{F}(X f), \mathcal{F}(g)\rangle_{\widehat{\mathcal{H}}}=\left\langle\mathcal{F}_{\alpha}(f), \mathcal{F}(g)\right\rangle_{2} .
$$

The density of $\mathcal{F}_{\alpha}(\mathcal{D})$ in $L_{2}^{G}$ completes the proof of (5.9).

Theorem 5.7. The operator $X$ is an isomorphism of $L_{2}^{G}$.

Proof. Since $\mathcal{D}$ is dense in $L_{2}^{G}$, it suffices to have the result on $\mathcal{D}$. From Lemma 5.5 and the formula (5.8), we have (see [6])

$$
\begin{aligned}
\|X(f)\|_{2}^{2}= & \|\mathcal{F} X(f)\|_{S}^{2}=\frac{1}{2 \pi} \int_{0}^{\infty}\left\|c(\lambda) G^{2}(\lambda) \mathcal{F}_{\alpha} f(\lambda)\right\|^{2} d \lambda \\
& \leq \frac{1}{2 \pi} \int_{0}^{\infty}\|c(\lambda) G(\lambda)\|^{2}\left\|G(\lambda) \mathcal{F}_{\alpha} f(\lambda)\right\|^{2} d \lambda
\end{aligned}
$$

From (3.10) we deduce that $\|c(\lambda) G(\lambda)\|$ is bounded and so there exists a positive constant $N_{1}$ such that

$$
\|c(\lambda) G(\lambda)\| \leq N_{1}
$$

Then we have

$$
\|X(f)\|_{2} \leq N_{1}\left\|\mathcal{F}_{\alpha}(f)\right\|_{2} \leq N_{1}\|f\|_{2} .
$$


Conversly, for $f$ in $\mathcal{D}$, using (5.8) we have

$$
\mathcal{F}_{\alpha} f(\lambda)=2 \pi G^{-2}(\lambda) S(\lambda) \mathcal{F} X f(\lambda) ;
$$

we deduce that

$$
\begin{gathered}
\|f\|_{2}^{2}=\|\mathcal{F} f\|_{S}^{2}=\left\|2 \pi G^{-2}(\lambda) S(\lambda) \mathcal{F} f(\lambda)\right\|_{2}^{2} \\
=\int_{0}^{\infty}\left\|2 \pi G^{-1}(\lambda) S(\lambda) \mathcal{F} X f(\lambda)\right\|^{2} d \lambda .
\end{gathered}
$$

Using again (3.10) we deduce that there exists a constant $N_{2}$ such that

$$
\|f\|_{2} \leq N_{2}\|\mathcal{F}(X f)\|_{S} .
$$

Finally the Plancherel formula allows us to deduce that

$$
N_{1}^{-1}\|X(f)\|_{\mathcal{H}} \leq\|f\|_{\mathcal{H}} \leq N_{2}\|X(f)\|_{\mathcal{H}}, \quad f \in \mathcal{D} .
$$

This suffices to conclude the theorem.

Remark 5.8. i) We define the generalized Fourier transform $\mathcal{F}$ and the transmutation operator $X$ on the space $\tilde{\mathcal{D}}$ of $C^{\infty}, M_{n}(\mathbb{C})$-valued functions, with compact support, respectively by the same expressions (4.1) and (5.5).

In particular Theorem 4.3, Propositions 5.3, 5.6 and Corollary 5.4 remain valid for these transformations.

ii) We denote by $\mathcal{L}_{2}$ the Hilbert space defined by

$$
\mathcal{L}_{2}=\{f:] 0, \infty\left[\rightarrow M_{n}(\mathbb{C})|| \| f||_{2}^{2}=\sum_{i, j=1}^{n} \int_{0}^{\infty} f_{i j}^{*}(u) u^{2 \alpha_{i}+1} f_{i j}(u) d u<\infty\right\} .
$$

Theorem 5.7 remains valid for this space.

\section{Generalized translations and Convolution}

Definition 6.1. i) We denote by $T_{y}, y \in \mathbb{R}$, the generalized translations operators, associated with $\left(\Delta_{\alpha}+q\right)$, and defined by

$$
T_{y} f=\mathcal{F}^{-1}\left[\mathcal{F}(f)(\lambda) \psi^{*}(\bar{\lambda}, y)\right], \quad f \in \tilde{\mathcal{D}} .
$$

ii) Let $f$ and $g$ be in $\tilde{\mathcal{D}}$. The generalized convolution product, associated with $\left(\Delta_{\alpha}+q\right)$, is the function $f \sharp g$ defined by

$$
f \sharp g=\mathcal{F}^{-1}[\mathcal{F}(f) \mathcal{F}(g)] .
$$

If we use (4.6) and (6.1), then the previous definition takes the form

$$
f \sharp g(x)=\int_{0}^{\infty} T_{y} f(x) G^{2}(y) g(y) d y .
$$

Remark 6.2. i) In the particular case when $q=0$ we denote by $\mathcal{T}_{x}$ (resp. $\star$ ) the generalized translation (resp. the convolution product) associated with $\Delta_{\alpha}$.

ii) When $g \in \tilde{\mathcal{D}}$ and $T \in \tilde{\mathcal{C}}_{*}^{\prime}$ (the space of even matrix-valued distributions with compact support), we can define $T \star g$ by

$$
T \star g=\mathcal{F}_{\alpha}^{-1}\left[\mathcal{F}_{\alpha}(T) \mathcal{F}_{\alpha}(g)\right] .
$$

It suffices to note that $\mathcal{F}_{\alpha}(T)$ belongs to the space of matrix-valued slowly increasing functions of exponential type.

The following properties derive immediately from Definition 6.1. 
Properties 6.3. For any $x \in \mathbb{R}$ and $f \in \tilde{\mathcal{D}}$, we have

- $T_{0} f(x)=f(x)$,

- $T_{x}(f) \in \tilde{\mathcal{D}}$

- $\left(\Delta_{\alpha}+q\right)_{x}^{*} T_{x} f=T_{x}\left(\Delta_{\alpha}+q\right) f$,

where

$$
\left(\Delta_{\alpha}+q\right)^{*} U(t)=U^{\prime \prime}(t)+U^{\prime}(t) \frac{2 \alpha+I}{t}+U(t) q^{*}(t) .
$$

Theorem 6.4. For any $f$ in $\tilde{\mathcal{D}},(x, y) \in \mathbb{R} \times \mathbb{R}$, the function $u(x, y):=T_{y} f(x)$ is the unique solution of the Cauchy problem (1.3), given by

$$
\left\{\begin{array}{l}
\left(\Delta_{\alpha}+q\right)_{x} u(x, y)=\left(\Delta_{\alpha}+q\right)_{y}^{*} u(x, y) \\
u(x, 0)=f(x),\left.\frac{\partial u}{\partial y}\right|_{(x, 0)}=0
\end{array}\right.
$$

Proof. For $f$ in $\tilde{\mathcal{D}}$, by definition, we have

$$
T_{y} f(x)=\int_{0}^{\infty} \psi(x, \lambda) S(\lambda) \mathcal{F}(f)(\lambda) \psi^{*}(y, \lambda) d \lambda+\sum_{j=1}^{m} \psi\left(x, \lambda_{j}\right) R_{j} S_{j} \hat{f}_{j} \psi^{*}\left(y, \lambda_{j}\right) ;
$$

then it is easy to see that $T_{y} f(x)$ is a solution of the problem (1.3). To have the unicity, we apply the Fourier transform, with respect to $x$, for the two members of the equation

$$
\left(\Delta_{\alpha}+q\right)_{x} u(x, y)=\left(\Delta_{\alpha}+q\right)_{y}^{*} u(x, y) .
$$

Then the function $v(\lambda, y)=\mathcal{F} u(., y)(\lambda)$ is a solution of the problem

$$
\left(\Delta_{\alpha}+q\right)_{y}^{*} v(\lambda, y)=\lambda^{2} v(\lambda, y)
$$

which is a differential equation of Fuchs type with a regular condition at zero. The unicity of the solution of a such system completes the proof.

The following proposition allows us to link the convolution products $\sharp$ and $\star$ by means of the transmutation operator $X$.

Proposition 6.5. For $f$ and $g$ in $\tilde{\mathcal{D}}$, we have

$$
\begin{aligned}
& (X f) \sharp g=X\left(f \star{ }^{t} X g\right), \\
& { }^{t} X(f \sharp g)={ }^{t} X(f) \star{ }^{t} X(g) .
\end{aligned}
$$

Proof. By the definition (6.2) we have

$$
(X f) \sharp g=\mathcal{F}^{-1}[\mathcal{F}(X f) \mathcal{F}(g)] .
$$

The formulas (5.8) and (5.9) yield that

$$
X f \sharp g=\mathcal{F}^{-1}\left[c^{*}(\lambda) c(\lambda) G^{2}(\lambda) \mathcal{F}_{\alpha}\left(f \star^{t} X g\right)(\lambda)\right] ;
$$

then we have (6.4) (from an idea of [1]).

Using the formulas (5.9) and (6.2), we have

$$
\begin{gathered}
\left(\mathcal{F}_{\alpha} \circ{ }^{t} X\right)(f \sharp g)=\left(\mathcal{F}_{\alpha} \circ{ }^{t} X\right)(f)\left(\mathcal{F}_{\alpha} \circ{ }^{t} X\right)(g) \\
=\mathcal{F}_{\alpha}\left[{ }^{t} X(f) \star{ }^{t} X(g)\right] ;
\end{gathered}
$$

the invertibility of $\mathcal{F}_{\alpha}$ allows us to deduce (6.5). 
In the proposition below we link the generalized translation operator $T_{x}$ and $\mathcal{T}_{x}$ by means of the transmutation operator $X$.

Proposition 6.6. For any $f$ in $\tilde{\mathcal{D}}$ we have

$$
T_{x} f(y)={ }^{t} X_{y}^{-1}\left[X_{x}\left(\mathcal{T}_{(.)}{ }^{t} X f\right)^{*}(y)\right]^{*} .
$$

Proof. We note that (see [21])

$$
\psi^{*}(\lambda, t)=\mathcal{F}_{\alpha}\left[K^{*}(t, .)\right](\lambda)
$$

here $K(t,$.$) denotes the distribution, in \mathcal{C}_{*}^{\prime}$, defined by $\langle K(t,), f\rangle=.X f(t)$, and $X f$ is given by the formula (5.5). Using Definition 6.1 we have

$$
\left[\mathcal{F}_{\alpha} \circ{ }^{t} X\right] T_{x} f=\mathcal{F}_{\alpha}\left[{ }^{t} X(f)\right] \mathcal{F}_{\alpha}\left[K^{*}(x, .)\right]=\mathcal{F}_{\alpha}\left[{ }^{t} X(f) \star K^{*}(x, .)\right],
$$

or equivalently

$$
T_{x} f(y)={ }^{t} X_{y}^{-1}\left[{ }^{t} X(f) \star K^{*}(x, .)\right](y) .
$$

From the formula (6.5) we have

$$
T_{x} f(y)={ }^{t} X_{y}^{-1} \int_{0}^{x}\left[\mathcal{T}_{u}\left({ }^{t} X f\right)(y)\right] G^{2}(u) K^{*}(x, u) d u,
$$

so we deduce the formula (6.6).

By use of Remark 5.8 and the previous proposition we have

Corollary 6.7. The mapping $f \rightarrow T_{y}(f), y \in \mathbb{R}$, extends to a continuous operator on $\mathcal{L}_{2}$.

\section{RiEmann's Method}

In this section we recover an integral representation for the solution of the Cauchy problem (1.3). Estimations on the solution and the kernel of its integral representation are given. This allows us to deduce a product formula for the regular eigenfunction associated with $\left(\Delta_{\alpha}+q\right)$. For this we use the Riemann method, and we suppose that $\alpha$ is a scalar matrix. Since the problem (1.3) has no symmetry in $x$ and $y$, Riemann's method leads us to study separately the cases $0<x \leq y$ and $0<y \leq x$. The Riemann function is used.

7.1. The Riemann function. Let $\Delta_{x y}$ denote the characteristic triangle in the $(s, t)$ plane with vertices $P(x, y), Q(x+y, 0)$ and $R(x-y, 0)$ (see Figure 1 ). B. L. J. Braaksma and H. S. V. De Snoo have studied in [2] the Riemann function in the domain $\Delta_{x y}$. Since, in the scalar case, the problem (1.3) is symmetric in $x$ and $y$, it is easy to extend the solution to the first quarter; this is not our case. A domain $\Omega$ of type Figure 2 is introduced to study the case $0<x \leq y$. Estimations on the Riemann function are established in each case. we The Riemann function $\mathcal{R}$ is defined as the unique solution $v(s, t)=\mathcal{R}(x, y ; s, t)$ of the characteristic boundary value problem

$$
\begin{cases}\left(L_{s}^{\alpha}-L_{t}^{\alpha}\right)^{* *} v(s, t)=0, & (s, t) \in \Delta_{x y} \\ v_{s}+v_{t}=\left(\alpha+\frac{1}{2}\right)\left(\frac{1}{s}+\frac{1}{t}\right) v, & s-t=x-y, \\ v_{s}-v_{t}=\left(\alpha+\frac{1}{2}\right)\left(\frac{1}{s}-\frac{1}{t}\right) v, & s+t=x+y, \\ v(x, y)=1 & \end{cases}
$$




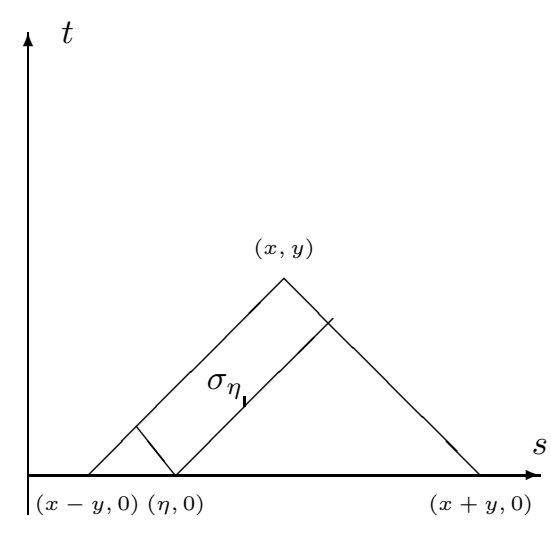

Figure 1 . The case $0<y \leq x$

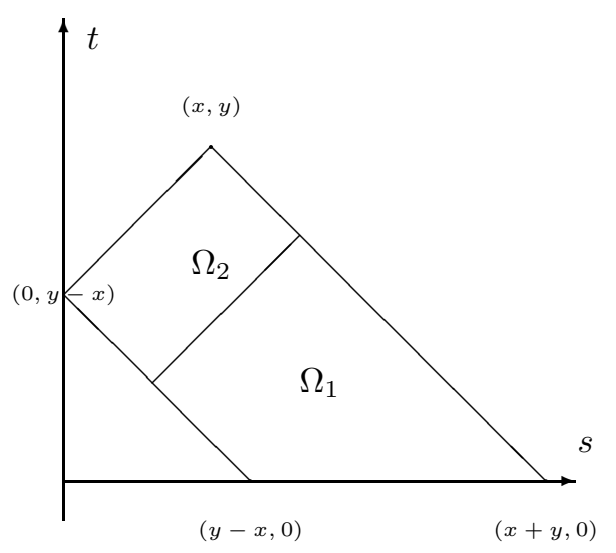

Figure 2. The case $0<x \leq y$

where $\left(L_{x}^{\alpha}-L_{y}^{\alpha}\right)^{* *}$ is the adjoint operator of $L_{x}^{\alpha}-L_{y}^{\alpha}$ and $L_{x}^{\alpha}$ is defined by

$$
L_{x}^{\alpha} u=u_{x x}+\frac{2 \alpha+1}{x} u_{x}, \alpha>-\frac{1}{2} .
$$

This problem is dealt with as the scalar case (see 9]) and (7.1) has a solution which is a diagonal matrix given by

$$
\mathcal{R}(x, y ; s, t)=\left(\frac{s t}{x y}\right)^{\alpha+\frac{1}{2}} P_{\alpha-\frac{1}{2}}(1-2 z) I,
$$

where $P_{l}$ denotes the $l$ th -Legendre function and

$$
z=\frac{\left\{(x-y)^{2}-(s-t)^{2}\right\}\left\{(x+y)^{2}-(s+t)^{2}\right\}}{16 x y s t} .
$$

- In the case $0<y \leq x$ we extend easily the results of [2] to have estimations on $\mathcal{R}$.

Let $R$ be function defined by

$$
R(x, y ; s, t)= \begin{cases}\frac{s t}{x y} & \text { if } \alpha \geq \frac{1}{2}, \\ \left(\frac{s t}{x y}\right)^{\alpha+\frac{1}{2}} Z^{\frac{1}{2}-|\alpha|} & \text { if } 0<|\alpha|<\frac{1}{2}, \\ \left(\frac{s t}{x y}\right)^{\frac{1}{2}} Z^{\frac{1}{2}}\left(1+\log Z^{-1}\right) & \text { if } \alpha=0\end{cases}
$$

where

$$
Z=16 x y s t\left\{(x+y)^{2}-(s-t)^{2}\right\}^{-1}\left\{(s+t)^{2}-(x-y)^{2}\right\}^{-1} .
$$

Then there exists a positive constant $M_{0}$ such that

$$
\|\mathcal{R}(x, y ; s, t)\| \leq M_{0} R(x, y ; s, t), \forall(s, t) \in \Delta_{x y} .
$$

Since $R(x, y ; s, t)$ is bounded on $\Delta_{x y}$, it follows that

$$
\|\mathcal{R}(x, y ; s, t)\| \leq M_{0}^{\prime}, \forall(s, t) \in \Delta_{x y} .
$$


- In the case $0<x \leq y$, one introduces the variables

$$
\begin{array}{ll}
2 \xi=y-x, & 2 \eta=y+x, \\
2 \xi_{0}=t-s, & 2 \eta_{0}=t+s .
\end{array}
$$

Instead of $z$ we use Chaundy's variables $x_{1}$ and $x_{2}$ (see [10]) defined as follows:

$$
x_{1}=\frac{(x+y-s-t)(y-x-t+s)}{4 x s}, \quad x_{2}=\frac{(x+y-s-t)(x-y-s+t)}{4 y t},
$$

so we have

$$
1-2 z=1-2 x_{1}-2 x_{2}-2 x_{1} x_{2}=1-2 \frac{\left(\xi_{0}^{2}-\xi^{2}\right)\left(\eta_{0}^{2}-\eta^{2}\right)}{\left(\eta^{2}-\xi^{2}\right)\left(\eta_{0}^{2}-\xi_{0}^{2}\right)} .
$$

The domain $\Omega$ is the union of the triangle $\Delta_{y x}$ and the rectangle $\Lambda_{x y}$ with vertices $(x, y),(0, y-x),(y-x, 0)$ and $(y, x)$.

When $(s, t)$ belongs to $\Lambda_{x y}$, we have

$$
0 \leq\left|\xi_{0}\right| \leq \xi \leq \eta_{0} \leq \eta<+\infty,
$$

and the formula (7.5) shows that $0 \leq z \leq 1$. We derive from the properties of $P_{l}$ (see [14]) that there exists a positive constant $M_{1}$ such that

$$
\left|P_{\alpha-\frac{1}{2}}(1-2 z)\right| \leq M_{1},(s, t) \in \Lambda_{x y} .
$$

When $(s, t)$ is in $\Delta_{y x}$ we remark that $x_{1}$ is positive while $x_{2}$ is negative, so the formula (7.5) shows that

$$
1 \leq 1-2 z \leq 1-2 x_{2}
$$

and on the other hand

$$
0 \leq-x_{2} \leq \frac{y}{t}
$$

so using the integral representation of $P_{l}$ we have

$$
\left|P_{l}(1-2 z)\right| \leq\left(1-2 x_{2}\right)^{l} \leq\left(\frac{6 y}{t}\right)^{l} .
$$

The previous estimates and the properties of the domain $\Omega$ yield

$$
\|\mathcal{R}(x, y ; s, t)\| \leq M_{1}^{\prime}\left(\frac{s}{x}\right)^{\alpha+\frac{1}{2}},(s, t) \in \Omega,
$$

where $M_{1}^{\prime}$ is a given positive constant.

Moreover, using the previous estimates, we have (see [2])

$$
\begin{aligned}
& \lim _{t \rightarrow 0^{+}} \mathcal{R}(x, y ; s, t)=0, \\
& \lim _{t \rightarrow 0^{+}} \frac{1}{2}\left[-\frac{\partial}{\partial t} \mathcal{R}(x, y ; s, t)+\frac{2 \alpha+1}{t} \mathcal{R}(x, y ; s, t)\right]=\omega_{0}(x, y, s) I,
\end{aligned}
$$

where

$$
\omega_{0}(x, y, s)=\frac{2^{1-2 \alpha} \Gamma(\alpha+1)}{\sqrt{\pi} \Gamma\left(\alpha+\frac{1}{2}\right)} s(x y)^{-2 \alpha} W^{\alpha-\frac{1}{2}}(x, y, s),
$$

with

$$
W(x, y, s)=\left\{(x+y)^{2}-s^{2}\right\}\left\{s^{2}-(x-y)^{2}\right\} .
$$


7.2. Solution in the case $0<y \leq x$. For any fixed point $(x, y) \in \mathbb{R}^{2}, 0<y \leq x$, and $(s, t) \in \Delta_{x y}$, let $v(s, t)=\mathcal{R}(x, y ; s, t)$ be the solution of $(7.1)$ and $u(s, t)$ the solution of the problem (1.3). We have

$$
\begin{array}{r}
v(s, t)\left[\left(L_{s}^{\alpha}-L_{t}^{\alpha}\right) u(s, t)\right]-\left[\left(L_{s}^{\alpha}-L_{t}^{\alpha}\right)^{* *} v(s, t)\right] u(s, t) \\
=u(s, t) q^{*}(t)-q(s) u(s, t) .
\end{array}
$$

We apply Green's theorem to the expression in the left on the triangle $\Delta_{x y}$ (see [7]); then we deduce that the function $u(s, t)$ is a solution of the integral equation

$$
u(x, y)=\int_{x-y}^{x+y} \omega_{0}(x, y, s) f(s) d s+\frac{1}{2} \int_{\Delta_{x y}} \mathcal{R}(x, y ; s, t)\left[q(s) u(s, t)-u(s, t) q^{*}(t)\right] d s d t .
$$

This integral equation is of Volterra type, and therefore can be solved by the successive approximations method. For this purpose we set

$$
u_{0}(x, y)=\int_{|x-y|}^{x+y} \omega_{0}(x, y, s) f(s) d s
$$

and, for $k \geq 0$,

$$
u_{k+1}(x, y)=\frac{1}{2} \int_{\Delta_{x y}} \mathcal{R}(x, y ; s, t)\left[q(s) u_{k}(s, t)-u_{k}(s, t) q^{*}(t)\right] d s d t .
$$

Theorem 7.1. Let $0<y \leq x$, and $f$ be a bounded measurable function. Suppose that there exists a measurable function $\mathcal{Q}(t)$ such that

$$
\|q(s)\|+\|q(t)\| \leq \mathcal{Q}(t), \quad s \geq t,
$$

and $\rho_{0}(y)=\int_{0}^{y} \mathcal{Q}(t) d t$ is finite; then the series

$$
u(x, y)=\sum_{k \geq 0} u_{k}(x, y)
$$

is uniformly convergent in any domain

$$
\Sigma_{\delta}=\left\{(x, y) \in \mathbb{R}^{2} \mid y \leq x, \text { and } 0<y \leq \delta\right\}, \delta<+\infty .
$$

The sum $u(x, y)$ is a solution of the problem (1.3), and there exist positive constants $M$ and $N$ such that

$$
\|u(x, y)\| \leq N\|f\|_{\infty} \exp \left\{M y \rho_{0}(y)\right\} .
$$

Proof. Using (7.8) and (7.11) we deduce that there exists a positive constant $N_{0}$ such that

$$
\left\|u_{0}(s, t)\right\| \leq N_{0}\|f\|_{\infty} .
$$

Then the hypothesis of the theorem, formulas (7.4) and (7.12) show recursively that

$$
\left\|u_{k}(s, t)\right\| \leq N_{0}\|f\|_{\infty} \frac{\left[M_{0}^{\prime} y \rho_{0}(y)\right]^{k}}{k !}, \quad k \geq 1,
$$

so Theorem 7.1 follows. 
7.3. The translation kernel. In this part we suppose that $0<y \leq x$ and that $q$ satisfies the hypothesis of Theorem 7.1.

Any $M_{n}(\mathbb{C})$-valued function $f$ can be written as

$$
f(x)=\sum_{1 \leq i, j \leq n} \delta^{i j}(x) f_{i j}(x),
$$

where $\delta^{i j}, 1 \leq i, j \leq n$, is the matrix-valued function defined by

$$
\delta_{k l}^{i j}= \begin{cases}1 & \text { if } \quad(k, l)=(i, j) \\ 0 & \text { if } \quad(k, l) \neq(i, j)\end{cases}
$$

and $f_{i j}$ is a scalar-valued function.

Let $i$ and $j \in \mathbb{N}, 1 \leq i, j \leq n$, be two fixed integers and consider the following Cauchy problem:

$$
\left\{\begin{array}{l}
u_{x x}+\frac{2 \alpha+1}{x} u_{x}-u_{y y}-\frac{2 \alpha+1}{y} u_{y}=u q^{*}(y)-q(x) u \\
u(x, 0)=\delta^{i j} h(x), \frac{\partial u}{\partial y}(x, 0)=0
\end{array}\right.
$$

where $h$ is a bounded, measurable and scalar-valued function.

Let $u(x, y)$ be the solution of (7.14). We shall show that each function $u_{k}(x, y)$, $k \geq 0$, in (7.12) may be represented by

$$
u_{k}(x, y)=\int_{x-y}^{x+y} w_{k}^{i j}(x, y, \eta) h(\eta) d \eta
$$

where, particularly, we have

$$
w_{0}^{i j}(x, y, \eta)=\omega_{0}(x, y, \eta) \delta^{i j}
$$

Let

$$
\tilde{w}^{i j}(x, y, \eta)=\sum_{k \geq 1} w_{k}^{i j}(x, y, \eta)
$$

then we have the following results.

Proposition 7.2. Under the hypothesis of Theorem 7.1 the series

$$
w^{i j}(x, y, \eta)=w_{0}^{i j}(x, y, \eta)+\tilde{w}^{i j}(x, y, \eta)
$$

is uniformly convergent on any compact region of $\Sigma_{\delta}$ and satisfies the estimates

1. $\left\|\tilde{w}^{i j}(x, y, \eta)\right\| \leq N \eta(x y)^{-1}\left[-1+\exp M \rho_{1}(y)\right], \frac{1}{2} \leq \alpha$,

2. $\left\|\tilde{w}^{i j}(x, y, \eta)\right\| \leq N \omega_{0}(x, y, \eta)\left[-1+\exp M \rho_{1}(y)\right], 0<\alpha<\frac{1}{2}$,

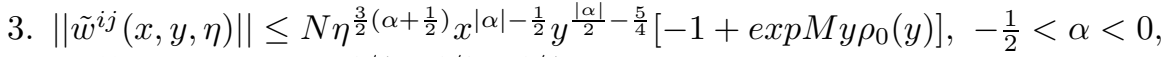

4. $\left\|\tilde{w}^{i j}(x, y, \eta)\right\| \leq N \eta^{3 / 4} x^{-1 / 2} y^{-1 / 4} \rho_{0}(y) \exp \left[M y \rho_{0}(y)\right], \alpha=0$,

where $N, M$ are positive constants depending on $\alpha, \rho_{\epsilon}(y)=\int_{0}^{y} t^{\epsilon} \mathcal{Q}(t) d t$, and $\epsilon>0$.

Proof. From (7.11) we obtain

$$
u_{0}(x, y)=\int_{x-y}^{x+y} w_{0}^{i j}(x, y, \eta) h(\eta) d \eta .
$$

Using the previous formula and changing the order of integration we rewrite (7.12), for $k=0$, in the form (see [15])

$$
u_{1}(x, y)=\frac{1}{2} \int_{x-y}^{x+y} \int_{\sigma_{\eta}} \mathcal{R}(x, y ; s, t)\left[q(s) w_{0}^{i j}(s, t, \eta)-w_{0}^{i j}(s, t, \eta) q^{*}(t)\right] h(\eta) d s d t d \eta
$$


where $\sigma_{\eta}$ is the rectangle with vertices $(x, y),\left(\frac{\eta+x-y}{2}, \frac{\eta-x+y}{2}\right),(\eta, 0)$ and $\left(\frac{\eta+x+y}{2}, \frac{x+y-\eta}{2}\right)$.

Recursively we have

$$
u_{k}(x, y)=\int_{x-y}^{x+y} w_{k}^{i j}(x, y, \eta) h(\eta) d \eta, k \geq 1
$$

with

$$
w_{k+1}^{i j}(x, y, \eta)=\frac{1}{2} \int_{\sigma_{\eta}} \mathcal{R}(x, y ; s, t)\left[q(s) w_{k}^{i j}(s, t, \eta)-w_{k}^{i j}(s, t, \eta) q^{*}(t)\right] d s d t .
$$

For the estimates of $w_{k}^{i j}(x, y, \eta)$ we use again the results of [2].

Since for $\alpha \geq \frac{1}{2}$ there exists a positive constant $M_{2}$ such that

$$
\left\|\mathcal{R}(x, y ; s, t) \omega_{0}(s, t, \eta)\right\| \leq M_{0} M_{2} \eta(x y)^{-1},(s, t) \in \Delta_{x y} .
$$

Hence by (7.16) we show, recursively, that

$$
\left\|w_{k}^{i j}(x, y, \eta)\right\| \leq M_{2} \eta(x y)^{-1} \frac{\left[M_{0} \rho_{1}(y)\right]^{k}}{k !}, k \geq 1 ;
$$

then we deduce (i).

In case of $0<\alpha<\frac{1}{2}$, we have $Z \leq 16 x y s t W^{-1}(x, y, \eta)$, so by (7.8) we have

$$
\left\|\omega_{0}(s, t, \eta) \mathcal{R}(x, y ; s, t)\right\| \leq M_{2} \eta(s t) W^{-1 / 2}(s, t, \eta) \omega_{0}(x, y, \eta) .
$$

Then, using the substitution $s^{2}=(\eta-t)^{2}+4 \eta t v$, we deduce that

$$
W(s, t, \eta)=16 t^{2} \eta^{2} v(1-v),
$$

and we show, recursively, that there exists a positive constant $M_{2}$ such that

$$
\left\|w_{k}^{i j}(x, y, \eta)\right\| \leq M_{2} \omega_{0}(x, y, \eta) \frac{\left[M_{0} \rho_{1}(y)\right]^{k}}{k !}, k \geq 1 ;
$$

from this we deduce (ii).

In the case $-\frac{1}{2}<\alpha<0$, according to (7.2) and (7.8), we have

$$
\left\|\omega_{0}(s, t, \eta) \mathcal{R}(x, y ; s, t)\right\| \leq M_{0} M_{2}(s t)^{-\alpha+\frac{1}{2}} \eta(x y)^{-\alpha-\frac{1}{2}} W^{\alpha-\frac{1}{2}}(s, t, \eta) ;
$$

using formulas (7.16) and (7.17), we show that

$$
\left\|w_{k}^{i j}(x, y, \eta)\right\| \leq M_{2} x^{|\alpha|-1 / 2} \eta^{\frac{3}{2}\left(\alpha+\frac{1}{2}\right)} y^{\frac{1}{2}|\alpha|-\frac{5}{4}} \frac{\left[M_{0} y \rho_{0}(y)\right]^{k}}{k !}, k \geq 1 .
$$

We discuss finally the case $\alpha=0$. Since we have $\omega_{0}(s, t, \eta)=M_{\alpha} \eta W^{-\frac{1}{2}}(s, t, \eta)$, using (7.2), we deduce that

$$
\left\|w_{1}^{i j}(x, y, \eta)\right\| \leq M_{0} M_{2} \eta(x y)^{-\frac{1}{2}} \int_{0}^{y} t^{\frac{1}{2}} \mathcal{Q}(t) \int_{\eta-t}^{\eta+t} s^{\frac{1}{2}} W^{-\frac{1}{2}}(s, t, \eta) d s d t .
$$

By the same substitution as the previous cases and thanks to (7.17) we deduce that

$$
\left\|w_{1}^{i j}(x, y, \eta)\right\| \leq M_{0} M_{2} \eta^{\frac{3}{4}}(x y)^{-\frac{1}{2}} \rho_{\frac{1}{4}}(y) .
$$

Then, recursively, we obtain

$$
\left\|w_{k}^{i j}(x, y, \eta)\right\| \leq M_{2} \eta^{\frac{3}{4}} x^{-\frac{1}{2}} y^{-\frac{1}{4}} y^{k-1} \frac{\left[M_{0} \rho_{0}(y)\right]^{k}}{k !} ;
$$

this completes the proof of the proposition. 
Theorem 7.3. In case of $0<y \leq x$ and under the hypothesis of Theorem 7.1 the function

$$
u(x, y)=\sum_{i, j=1}^{n} \int_{x-y}^{x+y} w^{i j}(x, y, \eta) f_{i j}(\eta) d \eta
$$

is the solution of the Cauchy problem (1.3).

7.4. Solution in the case $0<x \leq y$. In this case we introduce the domains $\Omega_{1}=\Omega \cap\{y \leq x\}$ and $\Omega_{2}=\Omega \cap\{x \leq y\}$ (see Figure 2, [8]). We apply again the Riemann method in the domain $\Omega$. After computation, we show that $\Omega$ is a characteristic domain in the same way as $\Delta_{x y}$. Indeed, for $v(s, t)=\mathcal{R}(x, y ; s, t)$, we have

$$
\begin{cases}\left(L_{s}^{\alpha}-L_{t}^{\alpha}\right)^{* *} v(s, t)=0, & (s, t) \in \Omega, \\ v_{s}+v_{t}=\left(\alpha+\frac{1}{2}\right)\left(\frac{1}{s}+\frac{1}{t}\right) v, & s-t=x-y, \\ v_{s}-v_{t}=\left(\alpha+\frac{1}{2}\right)\left(\frac{1}{s}-\frac{1}{t}\right) v, & s+t=x+y, \\ v_{s}-v_{t}=\left(\alpha+\frac{1}{2}\right)\left(\frac{1}{s}-\frac{1}{t}\right) v, & s+t=y-x, \\ v(x, y)=1 . & \end{cases}
$$

Considering the formula (7.10), applying Green's theorem to the expression in the left on the domain $\Omega$ and using (7.18), we show that the solution $U(x, y)$ of the Cauchy problem (1.3) satisfies, for $0<x \leq y$, the integral equation

$$
U(x, y)=u_{0}(x, y)+\frac{1}{2} \int_{\Omega} \mathcal{R}(x, y ; s, t)\left[q(s) U(s, t)-U(s, t) q^{*}(t)\right] d s d t
$$

where $u_{0}(x, y)$ is given by $(7.11)$.

Theorem 7.4. Let $0<x \leq y$; under the hypothesis of Theorem 7.1, the problem (1.3) has an unique solution $U(x, y)$ satisfying

$$
\|U(x, y)\| \leq N\|f\|_{\infty}\left(\frac{x+y}{2 x}\right)^{\alpha+\frac{1}{2}} \exp \left[2 M y \rho_{0}(y)\right]
$$

where $N$, and $M$ are given positive constants.

Proof. Let

$$
v_{0}(x, y)=\frac{1}{2} \int_{\Omega_{1}} \mathcal{R}(x, y ; s, t)\left[q(s) u(s, t)-u(s, t) q^{*}(t)\right] d s d t ;
$$

since, by Theorem $7.1, u(x, y)$ is completely defined on $\Omega_{1}$ so is $v_{0}(x, y)$, and by (7.6) we have

$$
\left\|v_{0}(x, y)\right\| \leq N\|f\|_{\infty} y \rho_{o}(y)\left(\frac{y+x}{x}\right)^{\alpha+\frac{1}{2}} \operatorname{expMy\rho _{0}(y).}
$$

Then we rewrite (7.19) in the form

$$
U(x, y)=u_{0}(x, y)+v_{0}(x, y)+\frac{1}{2} \int_{\Omega_{2}} \mathcal{R}(x, y ; s, t)\left[q(s) U(s, t)-U(s, t) q^{*}(t)\right] d s d t .
$$

Solving this problem causes us to consider the two following integral equations:

$$
\tilde{U}(x, y)=u_{0}(x, y)+\frac{1}{2} \int_{\Omega_{2}} \mathcal{R}(x, y ; s, t)\left[q(s) \tilde{U}(s, t)-\tilde{U}(s, t) q^{*}(t)\right] d s d t
$$

and

$$
\tilde{\tilde{U}}(x, y)=v_{0}(x, y)+\frac{1}{2} \int_{\Omega_{2}} \mathcal{R}(x, y ; s, t)\left[q(s) \tilde{\tilde{U}}(s, t)-\tilde{\tilde{U}}(s, t) q^{*}(t)\right] d s d t .
$$


To solve the previous integral equations we use successive approximations. By means of Theorem 7.1, the formulas (7.6) and (7.13) we deduce that there exists a positive constant $N_{1}$ such that

$$
\tilde{U}(x, y) \leq N_{1}\|f\|_{\infty}\left(\frac{y+x}{2 x}\right)^{\alpha+\frac{1}{2}} \exp \left[M y \rho_{0}(y)\right] .
$$

In a similar manner, using (7.6) and (7.20), we have

$$
\tilde{\tilde{U}}(x, y) \leq N_{1}\|f\|_{\infty}\left(\frac{y+x}{x}\right)^{\alpha+\frac{1}{2}} \exp \left[M y \rho_{0}(y)\right]\left[-1+\exp M y \rho_{0}(y)\right] .
$$

Then $\tilde{U}+\tilde{\tilde{U}}=U$ is a solution of (7.19), easily, we have Theorem 7 .

7.5. The product formula. The following result generalizes the product formula studied extensively in the scalar case (see [5], [7]).

Theorem 7.5. For any $\lambda \in \mathbb{C}$ we have

$$
\begin{aligned}
& \text { 1. } \psi(\lambda, x) \psi^{*}(\bar{\lambda}, y)=\sum_{1 \leq i, j \leq n} \int_{x-y}^{x+y} w^{i j}(x, y, \eta) \psi^{i j}(\lambda, \eta) d \eta, 0<y \leq x, \\
& \text { 2. } \psi(\lambda, x) \psi^{*}(\bar{\lambda}, y)=\sum_{1 \leq i, j \leq n} \int_{y-x}^{y+x}\left[w^{i j}(y, x, \eta)\right]^{*} \overline{\psi^{i j}(\bar{\lambda}, \eta)} d \eta, 0<x \leq y,
\end{aligned}
$$

where $w^{i j}(x, y, \eta)$ is given by the formula (7.15).

Proof. Theorem 3.5 and Corollary 3.3 show that, when $\lambda$ is real, the eigenfunction $\psi(\lambda, x)$ is bounded with respect to $x$ so we can apply the results of Theorem 7.3. Since

$$
\psi(\lambda, x)=\sum_{1 \leq i, j \leq n} \delta^{i j} \psi^{i j}(\lambda, x),
$$

the Cauchy problem (1.3), with initial conditions

$$
u(x, 0)=\psi(\lambda, x),
$$

has, when $0<y \leq x$, the following solution:

$$
u(x, y)=\sum_{1 \leq i, j \leq n} \int_{x-y}^{x+y} w^{i j}(x, y, \eta) \psi^{i j}(\lambda, \eta) d \eta, \quad \lambda \in \mathbb{R} .
$$

On the other hand $u(x, y)=\psi(\lambda, x) \psi^{*}(\bar{\lambda}, y)$ is a solution of the Cauchy problem (1.3) with the initial conditions (7.21). The unicity of the solution, and the analyticity of the function $\lambda \rightarrow \psi(\lambda, x)$ give us the theorem.

\section{REFERENCES}

[1] Yu. M. Berezanski, A. A. Kalyuzhnyi, Harmonic analysis in hypercomplex systems, Mathematical institute of the Academy of Sciences of Ukrainia, Kiev: Naukova Dumka, 1992 (Russian).

[2] B. L. J. Braaksma and H. S. V. De Snoo, Generalized translation operators associated with a singular differential operator, Proc. Conf. Ordinary and Partial Differential Equations, Dundee 1974 (B. D. Sleeman and I. M. Michael, eds.), Lecture Notes in Math., 415, SpringerVerlag, Berlin, 1974, pp.62-77. MR 54:10898

[3] R. Carroll, Transmutation, scattering theory and special functions, North-Holland Publishing Company, 1992.

[4] K. Chadan, P. C. Sabatier, Inverse problem in quantum scattering theory, Springer-Verlag, 1977. MR 58:25578 
[5] H. Chébli, Sur la positivité des opérateurs de "translation généralisée" associés à un opérateur de Sturm-Liouville sur ]0, $\infty$ [, C. R. Acad. Sci. Paris 275 (1972), 601-604. MR 58:6458

[6] H. Chebli, A. Fitouhi, M. M. Hamza, Expansion in series of Bessel functions for perturbed Bessel operators, J. Math. Anal. Appl. Vol. 181 (1994), 789-802. MR 95c:34004

[7] W. C. Connett, C. Markett and A. L. Schwartz, Convolution and hypergroup structures associated with a class of Sturm-Liouville systems, Trans. Amer. Math. Soc. 332 (1992), 365-390. MR 92c:34032

[8] E. T. Copson, On a singular boundary value problem for an equation of hyperbolic type, Arch. Rat. Mech. and Analysis 1 (1958), 349-356. MR 20:4080

[9] M. Coz and C. Coudray, The Riemann solution and the inverse quantum mechanical problem, J. Math. Phys., 17 (1976), 888-893. MR 58:1359

[10] M. Coz and P. Rochus, Partial differential equations for the inverse problem of scattering theory, J. Math. Phys., 17 (1976), 894-899. MR 55:5002

[11] N. Dunford and J. T. Schwartz, Linear Operators (part II): Spectral theory, John Wiley and Sons, 1963. MR 32:6181

[12] N. H. Fahem, Théorème de Paley-Wiener associé à un opérateur différentiel singulier à coefficients matriciels, C. R. Acad. Sci. Paris, 301 (1985) 821-823. MR 92a:33006

[13] A. Fitouhi, M. M. Hamza, A uniform expansion for the eigenfunctions of singular second order differential operators, SIAM J. Math. Anal. Appl. 21 (1990), 1619-1632. MR 92a:33006

[14] N. N. Lebedev, Special functions and their applications, Dover Publications, (1965). MR 30:4988

[15] B. M. Levitan, Generalized translation operators, Israel Program for Scientific Translations, Jerusalem, 1964. MR 30:2344

[16] J. L. Lions, Opérateurs de Délsarte et problèmes mixtes, Bull. Soc. Math. France, 84 (1956), 9-95. MR 19:556c

[17] N. H. Mahmoud, Théorème de Paley-Wiener associé à un opérateur différentiel singulier à coefficients matriciels, Thèse, Faculté des Sciences de Tunis (1985).

[18] N. H. Mahmoud, Differential operators with matrix coefficients and transmutations; Contemporary Mathematics (A.M.S), 183 (1995), 261-268. MR 96g:34004

[19] N. H. Mahmoud, Transmutation et translation généralisées associées à une famille d'opérateurs singuliers à coefficients matriciels, C. R. Acad. Sci. Paris 322 (1996), 525-528. MR 96m:35009

[20] R. G. Newton, Connection between the S-matrix and the tensor force, Phys. Rev., 100 (1955), 412-428. MR 17:619d

[21] K. Trimèche, Transformation intégrale de Weyl et théorème de Paley-Wiener associés à un opérateur différentiel singulier sur $(0, \infty)$, J. Math. Pures Appl. 60 (1981), 51-98. MR 83i:47058

[22] G. Watson, A treatise on the theory of Bessel functions, 2nd ed., Cambridge Univ. Press, London, New York, 1966. MR 96i:33010

Département de Mathématiques, Faculté des Sciences de Tunis, Campus Universitaire, 1060 Tunis, Tunisie

E-mail address: houda.mahmoud@insat.rnu.tn 